\title{
Analysis on Electromagnetic Force Properties of End Windings in Turbo-Generators
}

\author{
Guiji Tang ${ }^{1}$, Hongchun Jiang ${ }^{1}$, Yuling He ${ }^{1, ~}$, Gaurang Vakil ${ }^{2}$ \\ ${ }^{1}$ Hebei Key Laboratory of Electric Machinery Health Maintenance \& Failure Prevention, Department of Mechanical Engineering, North \\ China Electric Power University, Baoding, China \\ ${ }^{2}$ Department of Electrical and Electronics Engineering, University of Nottingham, Nottingham, UK
}

Email address:

heyuling1@163.com (Yuling He)

*Corresponding author

\section{To cite this article:}

Guiji Tang, Hongchun Jiang, Yuling He, Gaurang Vakil. Analysis on Electromagnetic Force Properties of End Windings in TurboGenerators. International Journal of Electrical Components and Energy Conversion. Vol. 6, No. 2, 2020, pp. 14-21.

doi: $10.11648 /$ j.ijecec.20200602.12

Received: December 14, 2020; Accepted: December 25, 2020; Published: December 31, 2020

\begin{abstract}
Electromagnetic forces on the end windings will stimulate intensive vibrations and cause insulation wearing, and the vibration wear will further induce the coil short circuit. Eventually accidents and losses will be brought. Therefore, it is of great significance to study the electromagnetic force properties of end windings. This paper studied on the electromagnetic force properties of end windings in turbo-generators. Firstly, the magnetic field in the end region was analyzed by winding MMF superposition principle and air gap magnetic conductivity method, and the electromagnetic force is got with Ampere law. Then, taking QFSN-600-2YHG turbo-generator as study object, the 3 directional electromagnetic forces and the mechanical responses on the end windings were calculate by electromagnetic-structure coupling finite element analysis method. Finally, the experiment vibrations are tested, and the acceleration data was compared with simulated result. It is shown that each directional electromagnetic force contains both the DC component and even harmonics, which will result in the winding vibration mainly at the double fundamental frequency. Meantime, the radial acceleration is the largest and the axial one is the least, so the radial vibration should be paid more attention to in daily monitoring. Moreover, the max deformation mainly occurs on the nose and middle part of the involute, and these locations should be strengthened in design and manufacture.
\end{abstract}

Keywords: Turbo-generator, End Winding, Electromagnetic Force, Vibration

\section{Introduction}

The stator winding of turbo-generator is a group of huge current carriers. During operation, the conductors in the end region will endure great electromagnetic forces, which will stimulate intensive vibrations and cause insulation wearing. Further, the vibration wear will induce the coil short circuit, and eventually lead to accidents and losses. Therefore, it is of great significance to study the electromagnetic force properties of end windings.

We can get the force on the end winding by the law of Ampere force, so the magnetic flux density (MFD) of the end zone is very important. G. K. M. Khan calculated the mirror image of windings and obtained the magnetic induction intensity of an arbitrary point in the end region according to
Biot-Safar law [1]. Yuda Hu presented the expression of the fundamental-frequency current density by Fourier series. And on this basis, detailed expressions of the magnetic induction strength in the end region were derived with the Maxwell equations [2]. However, the above two methods need accurate stator current information, and it is therefore limited for application in some complex running cases, for example, the winding inter-turn short circuit or rotor eccentricity. Yu-Ling $\mathrm{He}$ analyzed the stator and rotor vibration characteristics in eccentricity and inter-turn shortcircuit composite faults by magneto-motive force (MMF) superposition principle and air gap magnetic conductivity method $[3,4]$. Yucai Wu analyzed the virtual work during the rotor inter-turn short circuit [5]. Guoyuan Zhang and C. Patsios studied the end zone magnetic field and the unbalanced electromagnetic force of the rotor under eccentric 
fault respectively [6, 7]. And all of them can provide reference for the theoretical derivation of end winding's electromagnetic force.

In order to describe the force on the end winding and the exited response vividly, it is necessary to carry out a numerical simulation. It is found that the analytical calculation method is tedious and laborious, while the finite element simulation software is more suitable for the complex structure analysis of the end winding. The distribution of electromagnetic force on the end winding in normal condition, single-phase short circuit, two-phase short circuit, and three-phase short circuit case is analyzed [1, 8-10]. It is shown that the force density will decrease with the increasing of distance from the core, and the radial forces on the inner layer are stronger than those on the outer layer. Under the action of the electromagnetic forces, mechanical responses such as deformation, stress, elastic strain, etc., will be caused. The deformation (displacement) and stress distribution of the stator end winding in single-phase short circuit, double-phase short circuit, and three-phase short-circuited case are studied $[8,11,12]$. Yang Zhao employed the finite element software ABAQUS/CAE to obtain the transient magnetic force and it is found that the vibration mode of the end windings is ellipse [12]. Moreover, Hongbin Yin et al proposed an equivalent model of the stator-winding system for the quick modal analysis [13]. And T. Lugand carried out a comparing analysis of the stator vibration between the salient-pole synchronous machine and the doubly fed induction machine
[14].

However, by far, few of research has carried out the comprehensive study which includes not only the analytical derivation and the numerical simulation, but also the experimental validation. Because the force incentive is corresponding with the vibration response, so we can validate the theoretic result of the force by the simulated and experimental vibration data. This paper studies on the electromagnetic force on the end winding by employing the method illustrated in Figure 1. First, the electromagnetic force is deduced based on the analysis of the MFD and the winding current. Then, the electromagnetic force is calculated in Ansoft, and the mutual validation between the finite elementary calculated result and the theoretical conclusion is carried on. Thereafter, the mechanical responses of the end windings are calculated in ANSYS, and the simulated acceleration data can be obtained to verify the correction of the force result. Finally, the experiment is done to test the end winding vibration, and the comparing analysis with the simulated data can be conducted.

We have made some work about this topic [15]. As an extension of the pre-study, this paper carries out the experimental study for theoretical validation. In addition, the simulated mechanical response of acceleration is obtained for the comparison with the experiment data. Particularly, given that the vibration acceleration data is obtained in 3 directions, the end winding force components in this paper are obtained in radial, axial, and tangential directions.

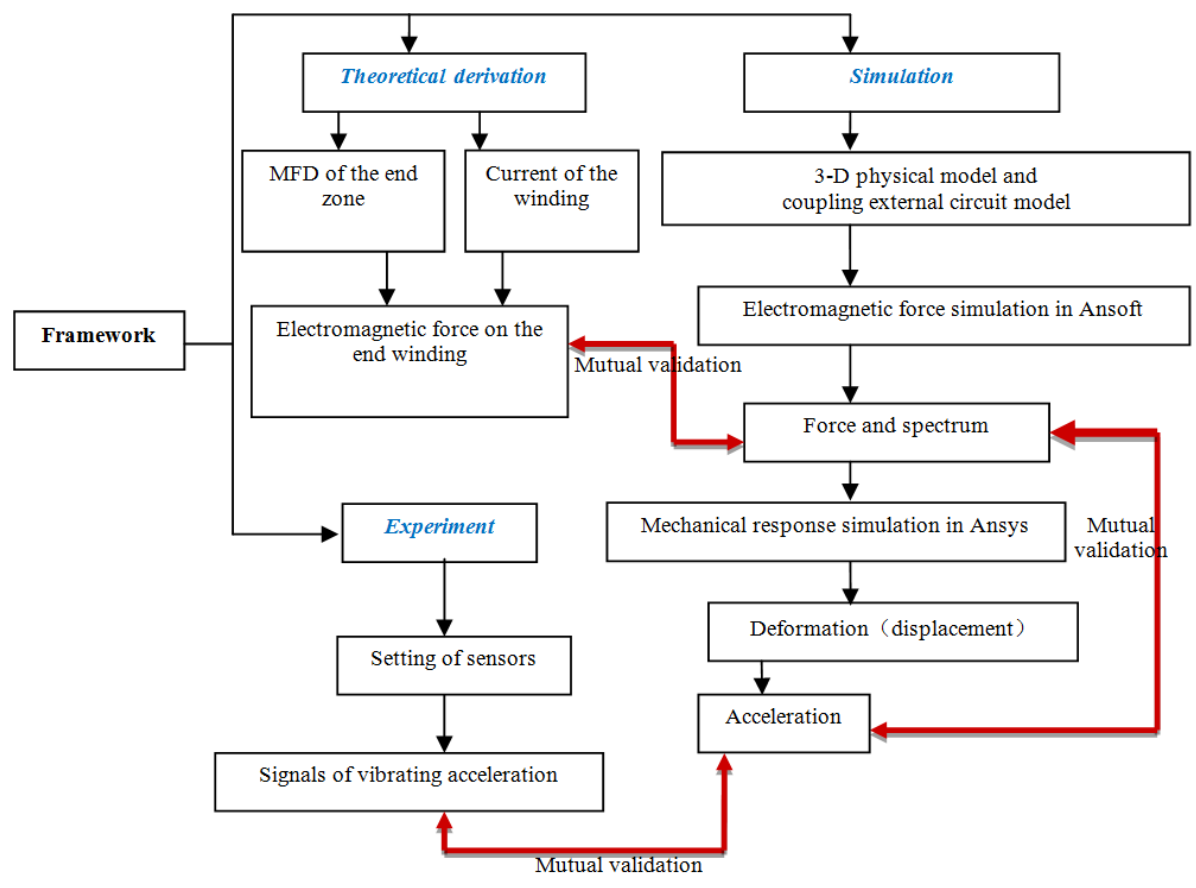

Figure 1. Analysis Framwork.

\section{Theoretical Analysis}

\subsection{Electromagnetic Force}

According to Ampere law, the force on the end part can be

written as

$$
\overline{F(t)}=\int_{l_{e}} \overline{B_{e}}(\alpha, t) \times \bar{I}(\alpha, t) d l_{e}
$$

Where $l_{\mathrm{e}}$ is the curve of the end, $B_{\mathrm{e}}$ and $I$ are the MFD and 
current of the end winding, respectively.

The magnetic motive force (MMF) at the air gap between stator and rotor can be considered as the superposition of stator winding MMF and rotor winding MMF, so the air gap MMF can be described as

$$
f(\alpha, t)=\sum_{n=1,3,5,7 \ldots}^{\infty}\left[F_{s n} \cos n(\omega t-p \alpha-\psi-0.5 \pi)+F_{r n} \cos n(\omega t-p \alpha)\right]
$$

Where $\alpha$ is the mechanical angle to indicate the circumferential position of the air gap, see Figure 2(b). $p$ is the number of the pole-pair and it is 1 for the study object in this paper, $\omega$ is the electrical angular frequency $(50 \mathrm{~Hz}), F_{s n}$ and $F_{r n}$ are the amplitudes of harmonic MMF of the stator and the rotor respectively.

The MFD is composed of the MMF and the pearmeance per unit area (PPUA), and it can be obtained by multiplying these two.

$$
B(\alpha, t)=f(\alpha, t) \Lambda_{0}
$$

Where $\Lambda_{0}$ is the PPUA $\left(\Lambda_{0}=\mu_{0} / g_{0}\right), g_{0}$ is the average value of the radial air-gap length between the stator core and the rotor core, and $\mu_{0}$ is the permeability of air/vacuum. Here $B$ is the MFD in the core zone, and the end zone MFD is the leakage of the core part. So we can depict $B_{\mathrm{e}}$ in (1) by adding a factor $f_{k}$

$$
B_{e}(\alpha, t)=f_{k} B(\alpha, t)
$$

Neglecting the influent of the windings' connection, according to law of electromagnetic induction, the current of winding can be written as

$$
I(\alpha, t)=B(\alpha, t) l v / z
$$

where $l$ and $Z$ are the length and impedance of the winding, respectively. The force on the end winding can be described as

$$
\left\{\begin{array}{l}
\overline{F(t)}=\int_{l_{e}} \overline{F_{k}(t)} d l_{e} \\
\overline{F_{k}(t)}=f_{k} \overline{B(\alpha, t)} \times \overline{i_{I}(\alpha, t)} d l_{e}=\left\{F_{k x}, F_{k y}, F_{k z}\right\} d l_{e}
\end{array}\right.
$$

Where $F_{\mathrm{k}}$ is the force density on an arbitrary point, $F_{\mathrm{kx}}$, $F_{\mathrm{ky}}$, and $F_{\mathrm{kz}}$ is the cartesian coordinate component in $\mathrm{X}, \mathrm{Y}$, and $\mathrm{Z}$ direction.

Further, we get the force and directional components on the whole end winding by integration as (7) and (8).

$$
\begin{gathered}
F(t)=\left(l v \Lambda_{0}^{2} / z\right) \int_{l_{e}} f_{k} f^{2}(\alpha, t) \sin \theta_{k} d l_{e} \\
\left\{\begin{array}{l}
F_{r}=\int_{l e}\left(F_{k x} \cos \theta+F_{k y} \sin \theta\right) d l_{e} \\
F_{t}=\int_{l e}\left(-F_{k x} \sin \theta+F_{k y} \cos \theta\right) d l_{e} \\
F_{a}=\int_{l e} F_{k z} d l_{e}
\end{array}\right.
\end{gathered}
$$

Where $\theta_{k}$ is the angle between the current vector and the MFD vector, $\theta$ is the vector angle of the arbitrary point, $F_{\mathrm{r}}, F_{\mathrm{t}}$ and $F_{\mathrm{a}}$ are the radial, tangential and axial force respectively. Equation (2) shows there are only odd harmonics such as $1^{\text {st }}$, $3^{\text {rd }}, 5^{\text {th }}$, and so on in the MMF, and it is indicated that the force is square of MMF in (7). So electromagnetic force in normal condition should contain even harmonics such as $2^{\text {nd }}, 4^{\text {th }}, 6^{\text {st }}$, et al.

\subsection{Vibration Response}

Deformation can be excited by the electromagnetic force and it is the displacement of vibration. Mathematically, the periodic movement can be expressed as a displacement array of the mass points, whose kinetic equation can be written as

$$
M \ddot{x}(t)+C \dot{x}(t)+K x(t)=F_{k}(t)
$$

Where $M$ is the mass matrix of the winding, $C$ is the damping matrix, $K$ is the stiffness matrix, and $F(t)$ is the exciting load matrix, i.e., the electromagnetic force matrix. $x(t), \dot{x}(t)$, and $\ddot{x}(t)$ are the displacement, velocity and acceleration response matrix, respectively. Since the displacement and the velocity are relatively tiny, so we simplify the above equation as (10). It is shown that the acceleration is in proportion to the force approximately.

$$
M \ddot{x}(t)=F_{k}(t)
$$

\section{Finete Element Simulation}

\subsection{Electromagnetic Force Simulation in Ansoft}

This paper takes QFSN-600-2YHG type turbo-generator as a study object and the 3D transient electromagnetic force of stator end windings is simulated in Ansoft. The structure of the generator is shown as Figure 2(a), and the core is as long as $6300 \mathrm{~mm}$. Therefore, the simulation process is very difficult, and program crash often occurs during gridding. So, how to simplify the physical model to save memory and ensure the smooth progress of simulation is the key point. The core length is set to $630 \mathrm{~mm}$ and the calculation of electromagnetic force was successfully completed in this paper. There are three types of grids for the model. The "length based" grid is used for rotor and stator cores, and the max length is set as $617.6 \mathrm{~mm}$. The same type of grid is used for rotor and stator windings, and the max length is set as $80 \mathrm{~mm}$ and $150 \mathrm{~mm}$ respectively. Meantime, the "surface approximation based" grid is also chosen for windings, considering the complexity of the structure. For the air gap between the rotor and stator cores, the "cylindrical gap based" grid is adopted because of the smallness of the space. The detailed physical model, coupling circuit model, and mesh message can be seen in the pre-study [15]. 


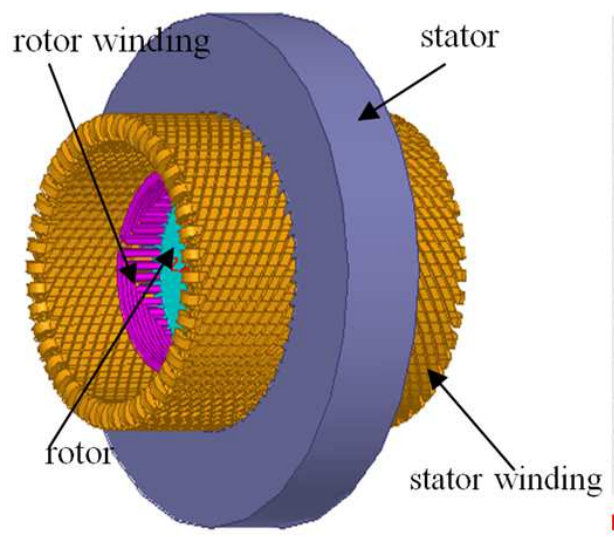

(a) 3D model

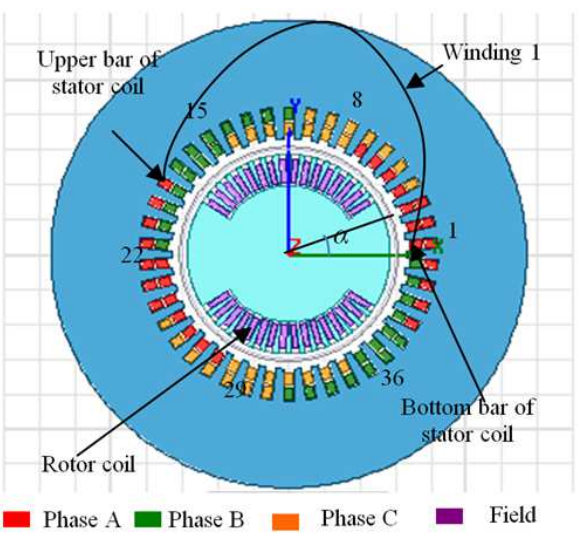

(b) Winding distribution section

Figure 2. Finete element model.

The electromagnetic force density and its cartesian coordinate components in $\mathrm{X}, \mathrm{Y}$ and $\mathrm{Z}$ direction can be obtained by simulation. In order to obtain the tangential, radial and axial electromagnetic force, it is necessary to use the field calculator for coordinate conversion and integral operation in the post-processing. The statement in the field calculator is shown in Figure 3. vfr, vft, and vfa represents directional force density in radial, tangential, and axial respectively.

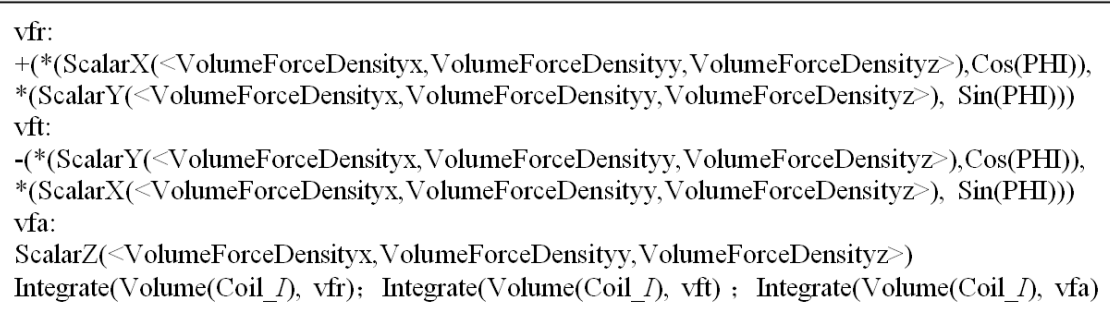

Figure 3. Staterments in field calculator.

The time domain wave and spectrum of the directional forces on end winding1 (see Figure 2(b)) is shown in Figure 4. It is shown that electromagnetic force contains mainly DC component and harmonics of $100 \mathrm{~Hz}\left(2^{\text {nd }}\right.$ harmonics $)$, and

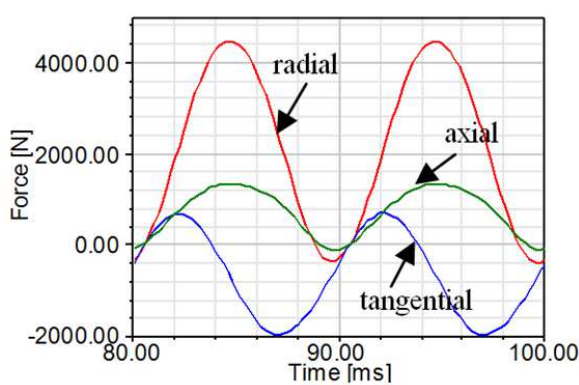

(a) Time domain force

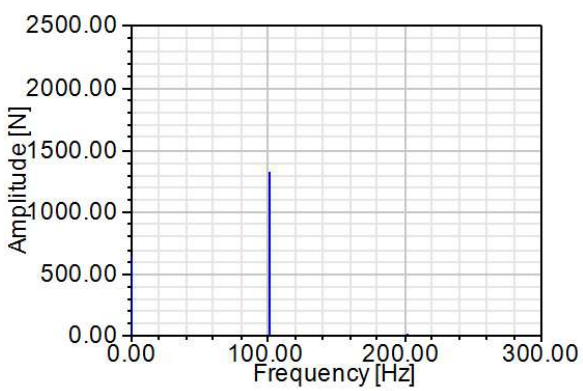

(c) Spectrum of tangential force this conclusion is consistent with theoretical analysis. Furthermore, the radial force amplitude is the largest and the axial one is the least. So, the end vibration in radial direction should be larger than others.

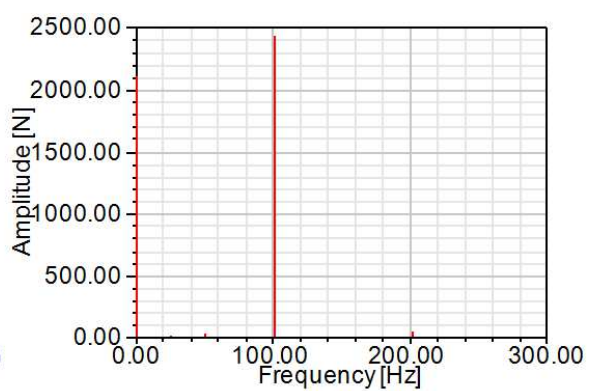

(b) Spectrum of radial force

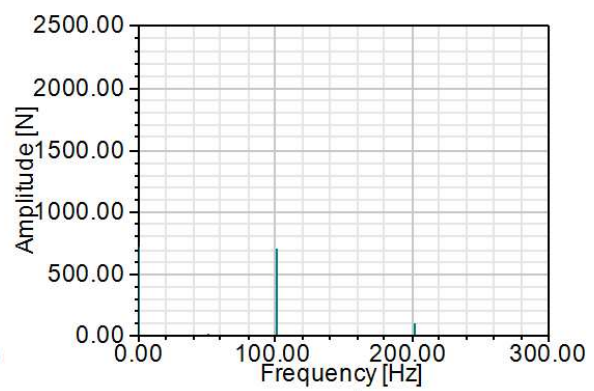

(d) Spectrum of axial force

Figure 4. Force on end winding 1. 


\subsection{Mechanical Response Simulation in Ansys}

To ensure the smooth coupling of electromagnetic force simulation and mechanical structural simulation, the model and transient electromagnetic force is imported into Ansys for finite element analysis. Deformation of Coil 1 is shown in Figure 5. We can see that the deformation distribution is

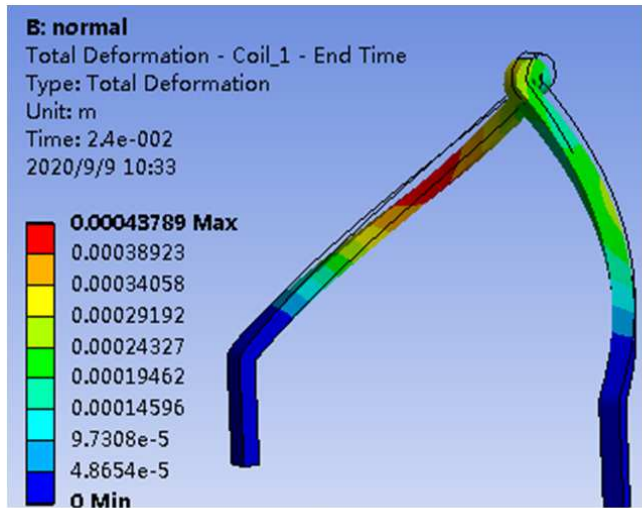

(a)

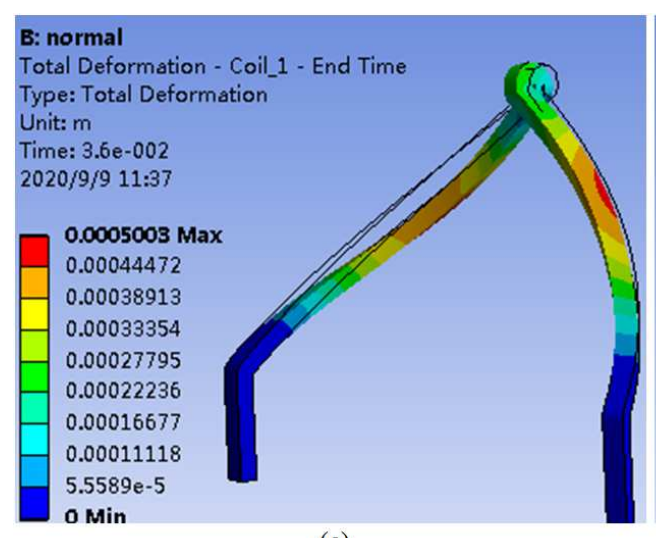

(c) varying with the time. Furthermore, the max deformation is located on the middle involute mainly, and on the nose part sometime. Therefore, these positions would endure more vibration and wearing, and should be strengthened in design and manufacturing.

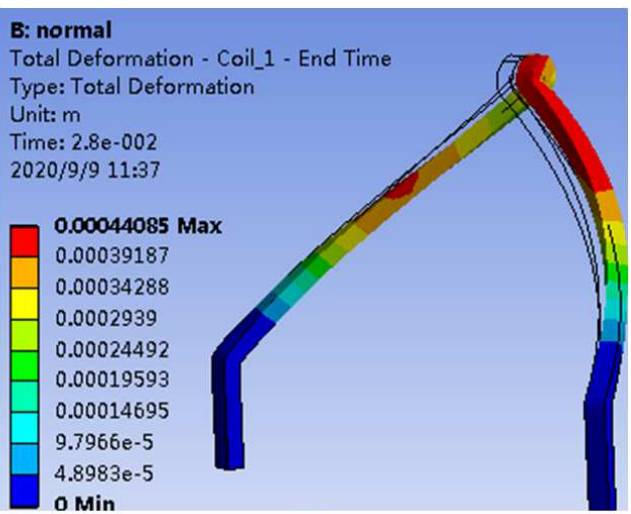

(b)

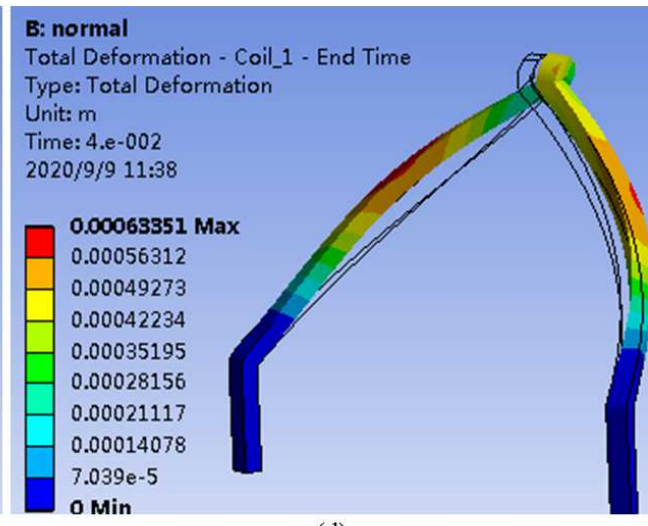

(d)

Figure 5. Maximum winding deformation of Coil 1 at: (a) left end, (b) nose top, (c) right end, and (d) both ends.

The time domain wave of deformation, velocity and acceleration is depicted in Figure 6 and Figure 7. It is indicated that the value of deformation and velocity is very small relative to acceleration. The order of magnitude is 1 and 100 for deformation and velocity respectively, but it is up to $10^{5}$ for acceleration, so it can explain the transformation of Eq. (9) to (10).

In order to mutual validate with the electromagnetic force,

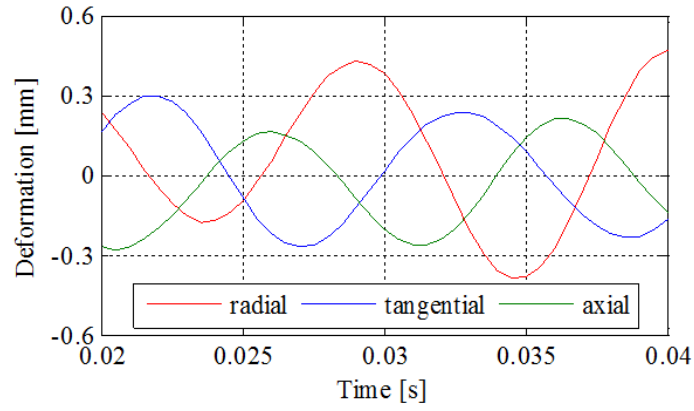

(a) deformation the acceleration is got on the middle point of the involute as shown in Figure 7. The acceleration contains obviously DC and $100 \mathrm{~Hz}$ harmonics. Moreover, the radial acceleration amplitude at $100 \mathrm{~Hz}$ is larger than the tangential one, and the axial one is the least. The results are consistent with the electromagnetic force simulation data in Figure 4 (b, c, d), and it reflects the corresponding relationship between incentive and response.

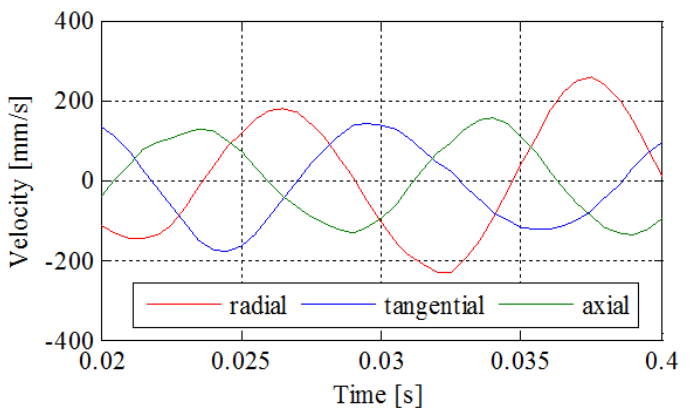

(b) velocity

Figure 6. Wave of deformation and velocity. 

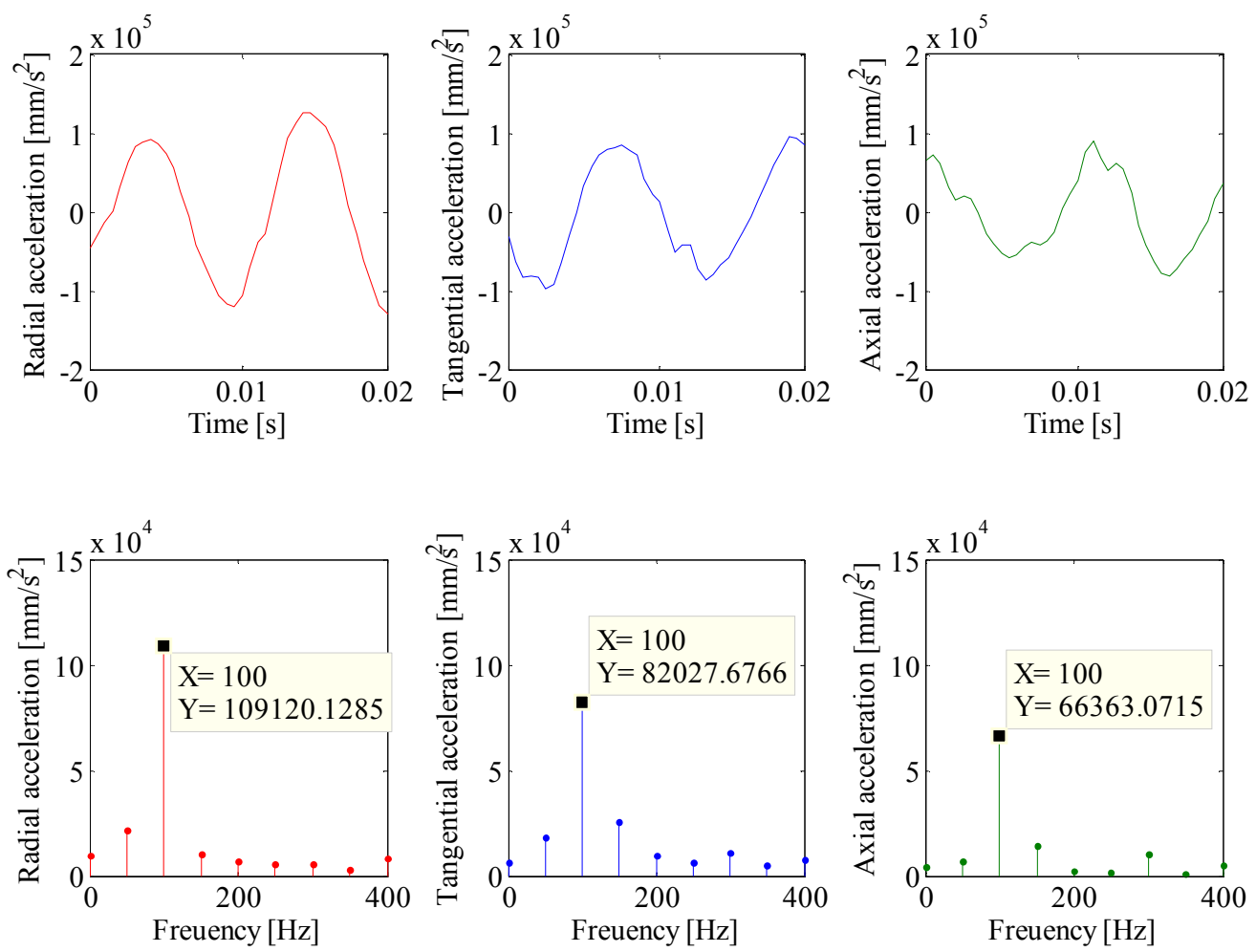

Figure 7. Acceleration wave and spectrum.

\section{Experimental Study}

In this paper, we employ the MJF-30-6 type prototype generator in the State Key Laboratory of Alternate Electrical Power systems with Renewable Energy Sources, P. R. China as the experiment object. This generator has 3 pairs of poles and its rated rotating speed is $1000 \mathrm{rpm}$. We fix 3 accelerometers to a same stator end winding. They are set in radial, tangential and axial direction, respectively, in order to acquire vibration signals in three directions, as shown in Figure 8.

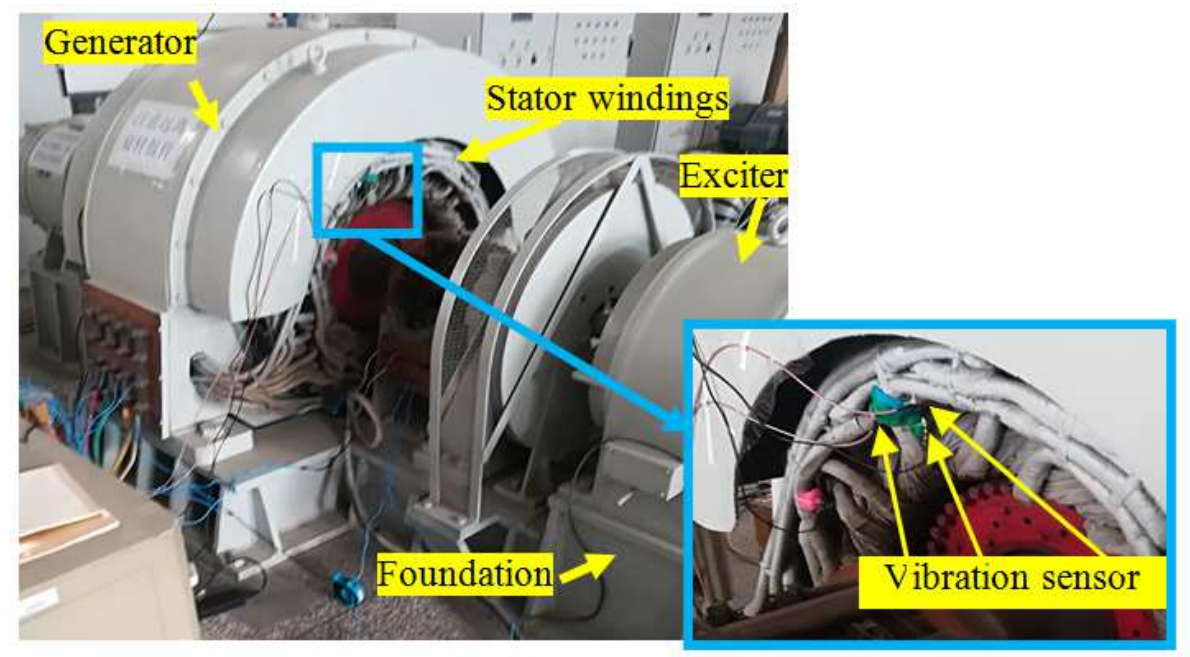

Figure 8. Experimental test setting

The tested vibration results are shown in Figure 9. It is indicated that the $2 \mathrm{nd}$ harmonic $(100 \mathrm{~Hz})$ have the prominent amplitude. The max amplitude at $100 \mathrm{~Hz}$ harmonics is in the radial direction and the min is in the axial. The result is consistent with the simulation, see Figure 7, and it validates the previous simulation. In daily monitoring, we should pay more attention to the radial vibration.
Moreover, the 1st harmonic $(50 \mathrm{~Hz})$ is also obvious and it is mainly transferred from the rotor, because the rotor rotates with the frequency of $16.7 \mathrm{~Hz}$ and there are 3 pairs of poles. For the axial vibration, there also exists obvious harmonics on $68.4 \mathrm{~Hz}$ and other components at times of $16.7 \mathrm{~Hz}$. The reason maybe that axial defect exits on the rotor. 

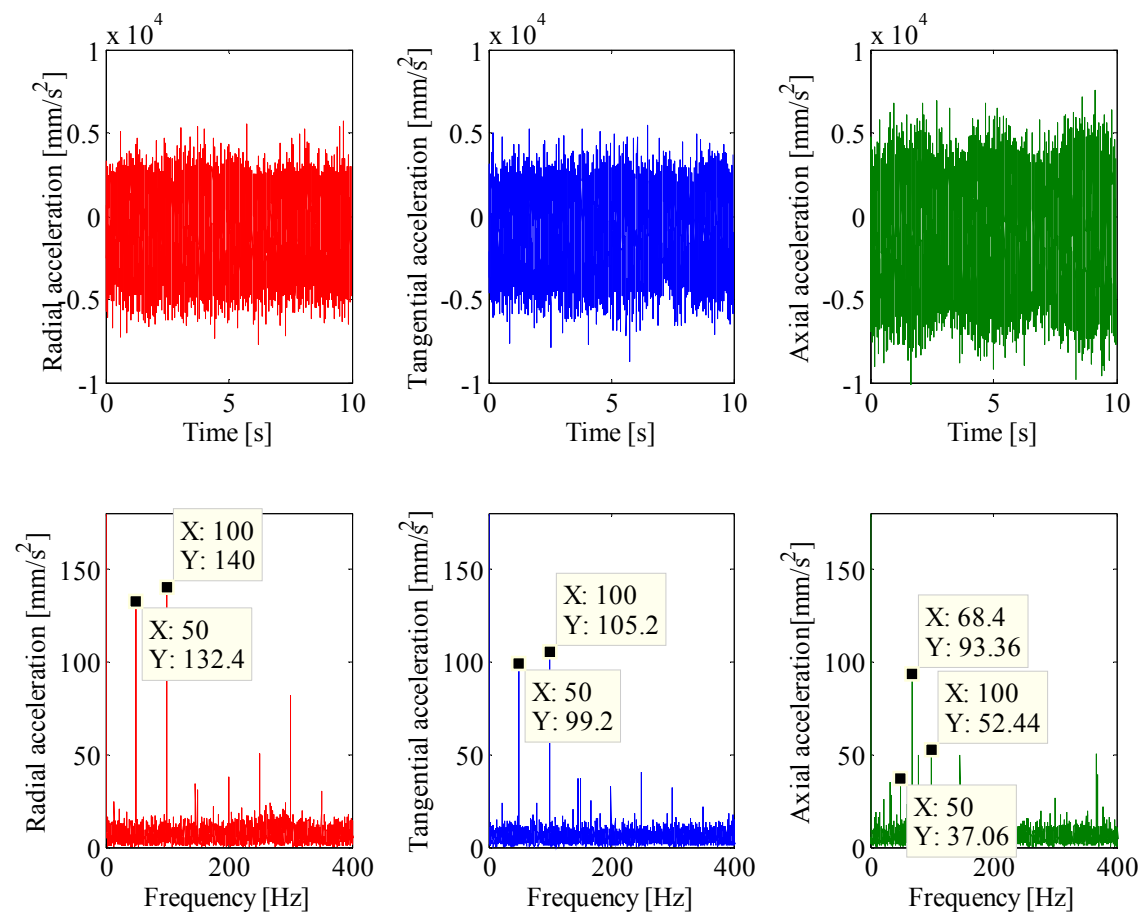

Figure 9. Experimental test vibration.

\section{Conclusions}

This paper investigates the electromagnetic force of the end wingding by theoretical analysis, simulation calculation and experiment study. The conclusions drawn up are list as follows.

First of all, each of the 3 directional electromagnetic forces contains DC component and even harmonics, which will result in the winding vibration mainly at the double fundamental frequency. Next, the acceleration response contains prevalent $100 \mathrm{HZ}$ harmonics and it is largest in radial direction. So the radial vibration should be paid more attention to in daily monitoring. Meantime, the experiment conclusion validates the simulation analysis results and it response the correspondence between incentive and response. Last, the max deformation mainly occurs on the nose and middle part of the involute, so the max vibration and wearing may happen on these positions. And these locations should be strengthened in design and manufacture.

The future work is to study the electromagnetic force properties of the end windings in cases of eccentricity and inter-turn short circuit. The electromagnetic force spectrum difference between normal and fault cases can be found, so it can provide an evidence for fault diagnosis. Moreover, the maximum deformation position and value can be obtained in fault cases, and these results are good reference for winding design and processing.

\section{Acknowledgements}

This work is supported by the National Natural Science Foundation of China (51777074), the Natural Science
Foundation of Hebei Province, China (E2020502032), the Chinese Fundamental Research Funds for the Central Universities (2017MS146, 2018YQ03), and the Hebei Provincial Top Youth Talent Support Program ([2018]-27).

\section{References}

[1] G. K. M. Khan, G. W. Buckley and N. Brooks, "Calculation of forces and stresses on generator end-windings. I. Forces," in IEEE Transactions on Energy Conversion, 19894 (4): 661670 .

[2] Hu Yuda, Qiu Jiajun, Qin Guanghui. "Electrtomanetic vibration of integrity end winding of large turbo-generator" in Proceedings of the CSEE, 07 (2003): 93-98 (in chinese).

[3] Yu-Ling He, Wei-Qi Deng, Bo Peng, etc., "Stator Vibration Characteristic Identification of Turbogenerator among Single and Composite Faults Composed of Static Air-Gap Eccentricity and Rotor Interturn Short Circuit," in Shock and Vibration, 2016, Open Access, DOI: 10.1155/2016/5971081.

[4] Yu-Ling He, Meng-Qiang Ke, Fa-Lin Wang, etc, "Effect of Static Eccentricity and Stator Inter-Turn Short Circuit Composite Fault on Rotor Vibration Characteristics of Generator," in Transactions of the Canadian Society for Mechanical Engineering, Vol. 39, No. 4, pp. 767-781, 2015.

[5] W. Yucai and L. Yonggang, "Diagnosis of Rotor Winding Interturn Short-Circuit in Turbine Generators Using Virtual Power," in IEEE Transactions on Energy Conversion, vol. 30, no. 1, pp. 183-188, March 2015.

[6] Zhang, Guoyuan, Wei, Junchao, Huang, Haizhou, Zhou, Miao, "A study on the nonlinear vibration of the generator rotor based on the unbalanced electromagnetic force and the oil film force coupling model," Journal of Vibroengineering, v 15, n 1, p 23-36, March 2013. 
[7] C. Patsios, A. Chaniotis, E. Tsampouris and A. Kladas, "Particular Electromagnetic Field Computation for Permanent Magnet Generator Wind Turbine Analysis," in IEEE Transactions on Magnetics, vol. 46, no. 8, pp. 2751-2754, Aug. 2010.

[8] R. Albanese et al., "Coupled Three Dimensional Numerical Calculation of Forces and Stresses on the End Windings of Large Turbo Generators via Integral Formulation," in IEEE Transactions on Magnetics, vol. 48, no. 2, pp. 875-878, Feb. 2012.

[9] Y. Fang, X. Bao, Q. 1v, X. Cheng and Y. He, "Analysis of Electromagnetic Force Distribution on End Winding of Electrical Submersible Motor During Starting Transient Operation," in IEEE Transactions on Magnetics, vol. 49, no. 10, pp. 5341-5345, Oct. 2013 (In the meantime, the electromagnetic force distribution of the winding was also investigated, showing that the radial forces on the inner layer are stronger than those on the outer layer).

[10] Liezheng Tang, Jiangjun Ruan, Hengyu Ding. "Analysis of Transient Magnetic Force on End-Winding in the Inverter-Fed Induction Machine," Journal of Electrical Engineering \& Technology, 2020, 15: 235-243.
[11] Stermecki, A., et al. "Numerical simulation of electromagnetic and mechanical phenomena in the end-winding region of three-phase induction machines*." E \& I Elektrotechnik Und Informationstechnik 128.5 (2011): 167-173.

[12] Y. Zhao, B. Yan, C. Zeng, S. Huang, C. Chen and J. Deng, "Optimal Scheme for Structural Design of Large Turbogenerator Stator End Winding," in IEEE Transactions on Energy Conversion, vol. 31, no. 4, pp. 1423-1432, Dec. 2016.

[13] H. Yin, X. Zhang, F. Ma, C. Gu, H. Gao and Y. Wang, "New Equivalent model and Modal Analysis of Stator Core-Winding System of Permanent Magnet Motor With Concentrated Winding," in IEEE Access, vol. 8, pp. 78140-78150, 2020.

[14] T. Lugand and A. Schwery, "Comparison Between the SalientPole Synchronous Machine and the Doubly Fed Induction Machine With Regard to Electromagnetic Parasitic Forces and Stator Vibrations," in IEEE Transactions on Industry Applications, vol. 53, no. 6, pp. 5284-5294, Nov.-Dec. 2017.

[15] Jiang H C, He Y L, Tang G J, etc., “A Comprehensive Analysis on Transient Electromagnetic Force Behavior of Stator Windings in Turbo-Generator," Mathematical Problems in Engineering, 2018, 2018: 1-16. 